\title{
Nêw-onset systemic lupus erythematosus in a pregnant woman: A case report
}

\author{
Mohadese Motaharinezhad ${ }^{1}$, Fatemeh Torklalebaq ${ }^{2}$, Maryam Moradi*1
}

1. Faculty of Nursing and Midwifery, Tehran University of Medical Sciences, Tehran, Iran (0000-0002-1941-6685)

2. Faculty of Nursing and Midwifery, Golestan University of Medical Sciences, Gorgan, Iran

\begin{abstract}
Background: Systemic lupus erythematosus (SLE) is a multi-systemic inflammatory disease that mainly affects women of childbearing age. Development of SLE during pregnancy increases the risk of some maternal and fetal complications. We herein present a pregnant woman with new-onset SLE during pregnancy.

Case description: A 30-year-old pregnant woman with a gestational age of 30 weeks and twin pregnancy was referred to a hospital because of bradycardia and arrhythmia of fetuses during routine pregnancy checkups. The patient was later diagnosed with SLE and received hydroxychloroquine and dexamethasone. During the course of treatment, the patient developed erythematosus skin lesions and was hospitalized for further evaluations. Various consultations were made. Given the overall conditions of the mother and fetuses, caesarean section was performed at week 34 of pregnancy. Finally, the patient was discharged with good general condition after one month of hospitalization.

Conclusion: Active SLE during pregnancy is associated with an increased risk of maternal and fetal complications. It is essential to consider the impact of pregnancy on the disease, the impact of the disease on fetal health and the safety of medications used during pregnancy and lactation. For improved fetal and maternal outcomes, a multidisciplinary approach comprising of gynecology, neonatology and internal medicine should be taken when treating pregnant women with SLE.
\end{abstract}

Keywords: Systemic lupus erythematosus; Pregnancy

Received: 2019/09/29 $\quad$ Revised:2019/10/05 $\quad$ Published:2019/10/25

*Correspondence: Department of Reproductive Health Research and Midwifery Consultation, Golestan University of Medical Sciences, Gorgan, Iran

(0000-0002-5191-510x)

Email: m.moradi@goums.ac.ir 


\section{INTRODUCTION}

Systemic lupus erythematosus (SLE) is a multi-systemic inflammatory disease that affects one in every 1,000 people (1). The is a disease of unknown etiology during which autoantibodies are produced, causing inflammatory damage in various organs including kidneys, central nervous system, etc. (2). A set of genetic, environmental and hormonal factors seem to be involved in the disease. In general, the prevalence of SLE is much higher in women, particularly during pregnancy, than in men. The onset of the disease is usually in the third or fourth decade of life (3). In recent decades, improvements in survival and quality of life of patients with SLE have led to an increase in the number of pregnancies during the course of the disease. Developing SLE during pregnancy increases the risk of some maternal and fetal complications (4). In addition to increased rate of recurrent miscarriage and intrauterine fetal demise (IUFD), SLE during pregnancy may lead to preterm labor, intrauterine growth restriction and prelabor rupture of membranes. Preeclampsia also appears to be one of the most common midwifery complications of women with SLE (5). On the other hand, safety of the drugs used for treatment of these patients is questionable.

Diagnostic criteria for SLE during pregnancy are not different from those for non-pregnant women (2). Ideally, the disease should be inactive 12-18 months or at least six months before pregnancy, which is accompanied with the best prognosis for both mother and fetus (6). Careful follow-up of pregnant women with SLE, management of complications and preventing administration of teratogenic drugs is essential.

Pregnancy is still a challenge for women with SLE. Concurrent management by gynecologists and rheumatologists could help improve pregnancy outcome. Pregnancy should be pre-planned and accompanied with special care and careful monitoring for women with lupus (7). Herein, we present a pregnant woman with new-onset SLE during pregnancy to provide a review of the most recent evaluation and management techniques for controlling the disease during pregnancy.

\section{CASE PRESENTATION}

A 30-year-old pregnant woman (G5,P3,L1,D2,Ab1) with gestational age of 30 weeks and twin pregnancy was referred to a hospital because of bradycardia and arrhythmia of fetuses during a routine pregnancy checkup one month ago. Complete heart block was confirmed via fetal echocardiography by a pediatric cardiologist. In later follow ups, SLE was confirmed by positive anti-RO and anti-nuclear antibody tests.

The patient was treated with hydroxychloroquine and dexamethasone for one month after the initial diagnosis of SLE and developed erythema and urticaria-like skin lesions approximately five days prior to admission, first in the upper limbs and then generalized to the trunk and lower extremities. The lesions were not itchy. After referral to a rheumatologist, the patient was hospitalized in the high risk pregnancy unit for suspected drug reaction. The patient had a previous history of IUFD and miscarriage, which was not investigated further to determine the etiology (Table 1). Moreover, the patient had no family history of SLE. 
Journal of Clinical and Basic Research (JCBR). 2019; 3(3): P 33-38.

Table 1. Records of previous pregnancies and childbirths

\begin{tabular}{|c|c|c|c|c|c|}
\hline Gravida & $\begin{array}{c}\text { Pregnancy } \\
\text { outcome }\end{array}$ & $\begin{array}{c}\text { Delivery } \\
\text { method }\end{array}$ & Gender & Time & $\begin{array}{c}\text { Gestational age } \\
\text { at birth }\end{array}$ \\
\hline G1 & Live & $\begin{array}{c}\text { Normal vaginal } \\
\text { delivery }\end{array}$ & Female & 10 years ago & Term \\
\hline G2 & Death & C-section & Male & 8 years ago & 8 months \\
\hline G3 & Abortion & $\begin{array}{c}\text { Dilation and } \\
\text { curettage }\end{array}$ & Male & 6 years ago & 4 months \\
\hline G4 & Death & C-section & Female & 2 years ago & 8 months \\
\hline
\end{tabular}

At birth, vital signs were controlled and normal. The patient's systolic and diastolic blood pressure was 110 and $60 \mathrm{mmHg}$, respectively. Except for generalized maculopapular erythematosus lesions, physical examination was normal and there was no sign of erythema, effusion or tenderness in the joints. The skin lesions were itchless, without scaling or $\mathrm{palm} / \mathrm{leg}$ involvement. There was no sign of vaginal bleeding and uterine contractility but the fetus was bradycardic. In addition to SLE medication, the patient was receiving insulin due to gestational diabetes mellitus. The patient's medications included hydroxychloroquine (200 mg daily), calciumD-glucaratedaily (daily), aspirin (80 mg daily), dexamethasone (2 $\mathrm{mg}$ B.D.), enoxaparin ampoule (4,000 units qhs), ferrous sulfate and folic acid. Results of daily paraclinical tests including complete blood count and liver and renal function tests were normal (Table 2).

\begin{tabular}{|c|l|}
\hline $\begin{array}{c}\text { Complete blood } \\
\text { count }\end{array}$ & White blood cell: $8.9 * 10^{3} / \mu 1$, Hemoglobin:11.7 g/dl, Hematocrit:33.3\%, Platelet: \\
\hline Liver function & $191 \mathrm{U} / 1$, Lactate dehydrogenase: $253 \mathrm{U} / \mathrm{l}$, Total bilirubin: $1.3 \mathrm{mg} / \mathrm{dl}$, Direct bilirubin: 0.4 \\
$\mathrm{mg} / \mathrm{dl}$
\end{tabular}

\section{Table 2. Results of the paraclinical tests}

Various consultations were requested for the patient. Perinatology consultation revealed no evidence of hydrops and pericardial effusion in the fetus and no need for betamethasone administration because of the dexamethasone treatment. Sonography and fetal echocardiography were performed. Fetal heart rate was measured for both fetuses every 6 hours. The hemodynamic and anatomical function of the twin on the right side were normal. The twin on the other side had sinus bradycardia due to the systemic disease of the mother; however, the pregnancy was continued due to the normal hemodynamic and cardiac function of the fetus.

Cardiology consultation was requested for the mother and fetuses and the results of echocardiographic evaluation of the mother 
were as follows: mild tricuspid regurgitation, normal right ventricle function, normal left ventricle size and function, ejection fraction $60 \%$ and pulmonary artery pressure 20 mmHg. However, both fetuses were bradycardic. Hemodynamic function of the heart was acceptable and there was no atrioventricular block in either case. Pericardial effusion was not evident. Fetal heart rate was abnormal (right twin: 81, left twin: 56). Moreover, the patient's blood glucose and insulin administration were adjusted.

The skin lesions (erythematosus plaques) formed throughout the body could be either due to active SLE or an adverse drug reaction to hydroxychloroquine. Ethacridine lactate solution and betamethasone topical were prescribed and hydroxychloroquine was discontinued. A skin biopsy was performed for the patient and the results indicated acute pustular eczema. During the course of treatment, the skin lesions improved significantly.

Laboratory and paraclinical examinations were performed frequently during the hospitalization period. The nonstress test was performed daily for both fetuses. Biophysical profile was performed twice weekly. Uteroplacental Doppler sonography was normal. Due to the patient's conditions, previous history of $\mathrm{C}$-section and reduction of amniotic fluid around the smaller fetus, the patient went under C-section at week 34 of pregnancy and two male babies with Apgar score of 8 were born. Prednisolone was administrated after caesarean delivery. Finally, the patient was discharged with good general condition after one month of hospitalization.

\section{DISCUSSION}

We reported a rare case of SLE during a twin pregnancy which was accompanied with fetal complications and complete heart block. According to studies, $90 \%$ of SLE cases are women aged 37 to 50 years (8). However, the age at onset in Iranian women is reported to be 21.5 years (9). Clinical and laboratory findings, course of the disease and prognosis vary from patient to patient (8). Common symptoms of SLE include fatigue, fever, skin lesions and renal involvement (7). In our case, the disease first presented with arrhythmia in the fetuses and later with skin manifestations. Despite advances made in the management of patients with SLE, the disease is still associated with a high risk of perinatal morbidity and mortality (10). In a study on 396 pregnant patients with SLE, 30\% of the patients experience a flare-up $18 \%$ of whom were affected by lupus nephritis (11). In another study on 51 patients with new-onset SLE during pregnancy, $90 \%$ of pregnancies were successful and 11 patients developed nephritis (12). Maternal renal disease, especially active lupus nephritis, renal dysfunction, serum creatinine $>2 \mathrm{mg} / \mathrm{dL}$, nephrotic syndrome, antiphospholipid syndrome and hypertension at the beginning of pregnancy are poor prognostic indicators for both mother and fetus. Patients with lupus nephritis, especially those with hypertension and antiphospholipid syndrome, are at risk of developing preeclampsia during pregnancy (6). A study has claimed that lupus nephritis is a risk factor for maternal outcomes such as flare-up, hypertension and anemia but has no significant relationship with adverse fetal outcomes (13). In our case, renal function test was normal and there was no evidence of renal involvement.

In SLE patients, renal function should be monitored by testing urinary protein excretion, urinary sediment analysis (hematuria, urinary cast), serum creatinine levels and glomerular filtration rate (14). Developing SLE during pregnancy considerably increases the risk of spontaneous abortion, IUFD, growth retardation and preterm delivery (1). Our case had a previous history of IUFD and spontaneous abortion, which had not been investigated.

Pregnant women with SLE should follow protocols of high risk pregnancies. Biometric findings and Doppler ultrasound in the third trimester and distinction between early and late IUGR will contribute to better decision making about delivery time in order to reduce the risk of perinatal morbidity and mortality 
(15). Indications of fetal echocardiography include fetal arrhythmia/dysrhythmia, myocarditis and positive maternal antiRO/SSA or anti-LA/SSB. Some studies also recommend hydroxychloroquine administration for women with SLE before and during pregnancy in order to control the disease and prevent flare-ups during pregnancy (16). Hydroxychloroquine may also reduce the risk of congenital heart block in fetuses exposed to maternal anti-Ro/SSA antibodies (17). In our case, after diagnosis of SLE, hydroxychloroquine therapy was initiated but later discontinued due to the patient's skin manifestations and the likelihood of an adverse drug reaction. Some studies suggest that the risk-benefit ratio of oral glucocorticoids, azathioprine, cyclosporine and tacrolimus is acceptable enough in controlling SLE in pregnancy (18). In moderate to severe cases, high-dose glucocorticoids (including intravenous pulse), intravenous immunoglobulin and plasmapheresis should be considered (19). In our case, oral and intravenous glucocorticoids were administered during the course of treatment. As recommended for the general population, calcium, vitamin $\mathrm{D}$ and folic acid supplements should be given to patients with SLE and antiphospholipid antibody syndrome, especially to those with low level of vitamin $\mathrm{D}$ in the first trimester of pregnancy and glucocorticoid and heparin users (6). Considering the conditions of pregnancy and long-term consumption of glucocorticoids and heparin, daily calcium supplementation was prescribed for our case to prevent the harmful effects of the medications on bone mass.

\section{CONCLUSION}

Systemic lupus erythematosus mainly affects women of childbearing age. Many factors may affect the pregnancy outcome and fetal complications of the disease. It is essential to consider the impact of pregnancy on the disease, effects of the disease on fetal health as well as the safety of medications used during pregnancy and lactation. Physicians should be aware of the physiological changes in pregnancy that mimic symptoms of SLE. For improved fetal and maternal outcomes, a multidisciplinary approach comprising of gynecology, neonatology and internal medicine should be taken when treating pregnant women with SLE.

\section{DECLARATIONS \\ Funding}

Not applicable.

Ethics approvals and consent to participate:

Oral consent was obtained from the subject and she was assured that her personal information will remain confidential.

\section{Conflict of interest}

The authors declare that there is no conflict of interest regarding the publication of this article.

\section{REFERENCES}

1. Smyth A, Oliveira GH, Lahr BD, Bailey KR, Norby SM, Garovic VD. A systematic review and meta-analysis of pregnancy outcomes in patients with systemic lupus erythematosus and lupus nephritis. Clinical Journal of the American Society of Nephrology. 2010;5(11):2060-8. [DOI:10.2215/CJN.00240110]

2. Jeremic K, Stefanovic A, Dotlic J, Stojnic J, Kadija S, Vilendecic Z, Janjic T, Jeremic J. Neonatal outcome in pregnant patients with antiphospholipid syndrome. Journal of perinatal medicine. 2015 ;43(6):761-8. [DOI:10.1515/jpm2014-0118]

3. Stojan G, Baer AN, Hochberg, Chakravarty, Clowse, Chakravarty, Moroni, Smyth, Buyon, Clowse, Huong. Flares of systemic lupus erythematosus during pregnancy and the puerperium: prevention, diagnosis and management. Expert review of clinical immunology. $2012 \quad ; 8(5): 439-53$. [DOI:10.1586/eci.12.36]

4. Yee CS, Akil M, Khamashta M, Bessant R, Kilding R, Giles I, Farewell VT, Gordon C. The BILAG2004-Pregnancy index is reliable for assessment of disease activity in pregnant SLE patients. Rheumatology. $2012 ; 51(10): 1877-80$. [DOI:10.1093/rheumatology/kes158]

5. Wu CQ, Kustec VE, Brown RN, Martin MC, Filion KB. The medical management of antiphospholipid syndrome in pregnancy: a meta- 
Journal of Clinical and Basic Research (JCBR). 2019; 3(3): P 33-38.

\begin{abstract}
analysis. Obstetrics \& Gynecology. 2014 ;123:178S-9S. [DOI:10.1097/01.AOG.0000447195.12926.b9]

6. Andreoli L, Bertsias GK, Agmon-Levin N, Brown S, Cervera R, Costedoat-Chalumeau N, Doria A, Fischer-Betz R, Forger F, Moraes-Fontes MF, Khamashta M. EULAR recommendations for women's health and the management of family planning, assisted reproduction, pregnancy and menopause in patients with systemic lupus erythematosus and/or antiphospholipid syndrome. Annals of the rheumatic diseases. 2017 ;76(3):476-85. [DOI:10.1136/annrheumdis-2016209770]
\end{abstract}

7. Nayana DH, Kondareddy T, Kaytri S. SLE during pregnancy, maternal and perinatal outcome in Teritary hospital. Int $\mathbf{J}$ Reprod Contracept Obstet Gynecol. 2017;6(2):507-11. [DOI:10.18203/2320-1770.ijrcog20170371]

8. Akbarian M, Rostamian A, editors. Systematic Lupus Erythmatosus. Tehran, Iran: Rheumatology Research Center, Tehran University of Medical Science; 2011. [Persian]

9. Akbarian M, Faezi ST, Gharibdoost F, Shahram F, Nadji A, Jamshidi AR, Akhlaghi M, Shafaie N, Akhlaghkhah M, Davatchi F. Systemic lupus erythematosus in Iran: a study of 2280 patients over 33 years. International journal of rheumatic diseases. 2010;13(4):374-9. [DOI:10.1111/j.1756185X.2010.01547.x]

10. Lateef A, Petri M. Systemic lupus erythematosus and pregnancy. Rheumatic Disease Clinics. 2017 ;43(2):215-26. [DOI:10.1016/j.rdc.2016.12.009]

11. Al Arfaj AS, Khalil N. Pregnancy outcome in 396 pregnancies in patients with SLE in Saudi Arabia. Lupus. 2010;19(14):1665-73. [DOI:10.1177/0961203310378669]

12. Carvalheiras G, Vita P, Marta S, Trovão R, Farinha F, Braga J, Rocha G, Almeida I, Marinho A, Mendonça T, Barbosa P. Pregnancy and systemic lupus erythematosus: review of clinical features and outcome of 51 pregnancies at a single institution. Clinical reviews in allergy \& immunology. 2010;38(2-3):302-6. [DOI:10.1007/s12016-009-8161-y]

13. Ku M, Guo S, Shang W, Li Q, Zeng R, Han M, Ge S, Xu G. Pregnancy outcomes in chinese patients with systemic lupus erythematosus (sle): a retrospective study of 109 pregnancies. PloS one. 2016;11(7):e0159364. [DOI:10.1371/journal.pone.0159364]

14. Bramham K, Hunt BJ, Bewley S, Germain S, Calatayud I, Khamashta MA, Nelson-Piercy C.
Pregnancy outcomes in systemic lupus erythematosus with and without previous nephritis. The Journal of rheumatology. 2011;38(9):1906-13.

[DOI:10.3899/jrheum.100997]

15. Sovio U, White IR, Dacey A, Pasupathy D, Smith GC. Screening for fetal growth restriction with universal third trimester ultrasonography in nulliparous women in the Pregnancy Outcome Prediction (POP) study: a prospective cohort study. The Lancet. 2015;386(10008):2089-97. [DOI:10.1016/S0140-6736(15)00131-2]

16. Koh JH, Ko HS, Kwok SK, Ju JH, Park SH. Hydroxychloroquine and pregnancy on lupus flares in Korean patients with systemic lupus erythematosus. Lupus. 2015;24(2):210-7. [DOI:10.1177/0961203314555352]

17. Tunks RD, Clowse ME, Miller SG, Brancazio LR, Barker PC. Maternal autoantibody levels in congenital heart block and potential prophylaxis with antiinflammatory agents. American journal of obstetrics and gynecology. 2013;208(1):64-e1. [DOI:10.1016/j.ajog.2012.09.020]

18. Webster P, Wardle A, Bramham K, Webster L, Nelson-Piercy C, Lightstone L. Tacrolimus is an effective treatment for lupus nephritis in pregnancy. Lupus. 2014;23(11):1192-6. [DOI:10.1177/0961203314540353]

19. Skorpen CG, Hoeltzenbein M, Tincani A, Fischer-Betz R, Elefant E, Chambers C, Da Silva J, Nelson-Piercy C, Cetin I, Costedoat-Chalumeau N, Dolhain R. The EULAR points to consider for use of antirheumatic drugs before pregnancy, and during pregnancy and lactation. Annals of the rheumatic diseases. 2016;75(5):795-810. [DOI:10.1136/annrheumdis-2015-208840] 